\title{
RECURSOS DE ATENUACIÓN EN LA 'ORIENTACIÓN' DE NARRATIVAS PERSONALES ORALES DESDE UNA PERSPECTIVA SOCIOPRAGMÁTICA ${ }^{1}$ \\ MITIGATION RESOURCES FOUND IN THE ORIENTATION OF ORAL PERSONAL NARRATIONS BASED ON A SOCIOPRAGMATICA PERSPECTIVE
}

\author{
Javier González Riffo \\ Pontificia Universidad Católica de Chile, Chile \\ jdgonzalez@uc.cl \\ Silvana Guerrero González \\ Universidad de Chile, Chile \\ siguerrero@u.uchile.cl
}

\begin{abstract}
Resumen:
En esta investigación se indaga en el comportamiento sociopragmático de los recursos de atenuación presentes en la 'orientación' de narrativas personales orales. Se trabaja con base en 90 narraciones, 36 individuales y 54 co-construidas (o conversacionales), de hablantes de Santiago de Chile, estratificados por sexo y grupo socioeconómico. Los principales hallazgos de este estudio indican que los recursos de atenuación presentes en la 'orientación' de relatos orales corresponden a 'desfocalizadores'. Asimismo, se concluye que dichos recursos son más recurrentes en narraciones individuales antes que en las conversacionales. Se aprecia, además, que son las mujeres las que utilizan en mayor medida recursos de atenuación en las historias narradas individualmente y, finalmente, se puede concluir que el empleo de estos recursos aumenta conforme se asciende en el grupo socioeconómico de los hablantes.
\end{abstract}

Palabras clave: narraciones orales de experiencia personal, orientación extratemática, recursos de atenuación, desfocalizadores.

\begin{abstract}
:
This investigation delves into the sociopragmatic behavior of the mitigation resources found in the 'orientation' of oral personal narrations. It is based on 90 narrations, of which 36 were individual and 54 were co-constructed (or conversational), of Santiago de Chile speakers, stratified by sex and socioeconomic segment. The main findings of this study show that the mitigation resources found in the 'orientation' of oral personal narrations correspond to 'defocalizers'. Furthermore, it was concluded that the aforementioned resources are more frequent in individual narrations rather than in conversational narrations. It was also observed that females are the ones that employ mitigation resources the most in stories narrated individually. Finally, it was also concluded that the use of these resources increases just as the socioeconomic segment of the speakers rises.
\end{abstract}

Keywords: oral narrations of personal experiences, extrathematic orientation, mitigation resources, defocalizers.

Recibido: 31 de marzo de 2018

Aceptado: 4 de junio de 2018

\footnotetext{
${ }^{1}$ La presente investigación cuyo propósito es estudiar la co-construcción de narraciones de experiencia personal en relación con los factores génerolectal y sociolectal, en una muestra de hablantes de Santiago de Chile.

Se enmarca en el Proyecto FONDECYT 11150007,
} 


\section{Introducción}

Ya desde los presupuestos teóricos de Bassols y Torrent (2003) se sugiere que toda narración contiene un proceso orientado y complicado, es decir, toda narración incluye una sucesión mínima de eventos que se caracterizan por su orientación hacia un final y su complicación. Según las autoras, la orientación hacia un final implica un carácter temporal y una integración. No obstante, el orden de los acontecimientos del relato puede diferir del orden de los acontecimientos de la historia en la medida en que presenten unidad de acción y se orienten hacia un final.

Con base en los presupuestos teórico-metodológicos de la sociolingüística variacionista e interaccional, en esta investigación se estudia el empleo de recursos de atenuación presentes en la 'orientación' de 90 narraciones de experiencia personal generadas por una muestra estratificada de hablantes de Santiago de Chile. 36 de las narraciones que conforman la muestra de estudio han sido generadas de manera individual en el marco de entrevistas sociolinguiísticas, mientras que las 54 narrativas restantes se obtienen mediante la aplicación de un diseño narrativo a parejas de hablantes de la mencionada comunidad de habla. La muestra ha sido estratificada según las variables sexo y grupo socioeconómico. De manera específica, se relaciona la presencia de recursos de atenuación con los recursos de orientación extratemática (Guerrero, 2014b), se determina la sensibilidad a la variación de los recursos de atenuación en orientación narrativa según el tipo de narración, individual o conversacional, y, por último, se compara el uso de este fenómeno en relación con los factores sexo y grupo socioeconómico de los hablantes.

Las metas propuestas aportan a los estudios sociolingüísticos en general, pero más particularmente a aquellos que se centran en la variedad chilena del español y a la discusión de los métodos de elicitación de corpus. Asimismo, aportamos a la descripción del fenómeno de la atenuación desde una perspectiva sociopragmática, que permita referir también a cómo los hablantes construyen, comparten y negocian su identidad en la interacción con base en la idea de compromiso discursivo. 


\section{Constructo teórico}

2.1. Sobre las narrativas orales de experiencia personal y su estudio en la sociolingüística

Como ya ha precisado Guerrero (m.i.), narración, relato e historia pueden aplicarse al estudio de la narrativa oral desde la perspectiva sociolingüística (cf. Norrick, 2000; Riessman, 2008; Guerrero, 2017a), de manera indistinta. Esta investigación se refiere a narraciones o relatos de experiencia personal que deben ser dignas de contarse, o, en otras palabras, ser reportables (Labov, 2004) y deben constituir también narraciones sobre hechos que se supone ocurrieron realmente (Labov, 1972: 360 y ss.; Labov, 2013: 21).

Los estudios sobre narrativas en sociolingüística han comprobado que las narraciones son formas privilegiadas de discurso que desempeñan un papel central en casi todas las conversaciones, y se trata, además, en su versión prototípica, de un ejemplo de evento de habla bien formado, que contiene un principio, un medio y un fin (Labov, 1997).

En lo que respecta al enfoque sociolingüístico, las investigaciones sobre narrativas han construido las muestras de estudio sobre la base de relatos que se producen en el contexto de una entrevista sociolingüística, en cuya situación el entrevistador constituye un público ideal (Labov, 1997). No obstante, son muchas las críticas hacia este enfoque. Norrick (2000) y Juzwik (2012) señalan que Labov (1972) se concentra en narrativas completas, orales, de experiencia personal desde la perspectiva del entrevistado, pero no considera los efectos de la interacción. También Thornborrow (2012) destaca que la narración es un fenómeno interactivo y que Labov no solo no trata con el discurso narrativo contextualmente situado, sino que además no presta atención a aspectos de la interacción. El mismo énfasis es otorgado por De Fina y Johnstone (2015), quienes precisan que Labov solo se concentra en la producción de cláusulas del narrador, y no considera las contribuciones de los demás participantes ni del contexto interaccional. 


\subsection{Sobre la ‘orientación’ narrativa}

Labov (1972, 2013) y Labov y Waletzky (1967) proponen que una narración de experiencia personal plenamente formada exhibe seis rasgos estructurales claramente definidos, a saber, el resumen, la orientación, la complicación de la acción, la evaluación, la resolución y la coda. El rasgo que consideramos fundamental para llevar a cabo investigación sobre la variación discursiva es la orientación, entendida como aquel segmento de la narración que hace referencia a un tiempo, un lugar, unas personas y una conducta esperada en cierta situación (Labov, 2013).

Soler (2004), tomando como referencia el trabajo de Johnstone (1990), atribuye categorías más específicas a la propuesta laboviana (Guerrero, 2014b). Concretamente, la autora alude a la especificación de lugar, la especificación de tiempo, la descripción de personajes, los nombres de lugares y los nombres de personas. No obstante, para Guerrero (2014b) estos elementos no son suficientes para dar cuenta de todas las categorías orientacionales que se explicitan en las narrativas de experiencia personal, lo que la lleva a proponer nueve categorías de orientación extratemática, derivas de un trabajo inductivo, que partió de la revisión de 192 narraciones de experiencia personal del español de Santiago de Chile. Dichas categorías son: especificación de tiempo, especificación de lugar, nombres propios de lugares, descripción objetiva de lugares, especificación de personajes, nombres propios de personajes, descripción objetiva de personajes, descripción de situaciones y descripción de objetos.

Como sugiere Guerrero (m.i.), el estudio de la orientación narrativa es fundamental toda vez que se consideran los postulados de Johnstone (2001; 2006), quien pone de relieve que la estructura y la función de la narrativa, en tanto proceso de construcción y narración resultante, pueden contribuir a la descripción de la lengua vernácula, puesto que podrían relacionarse con conjuntos particulares de rasgos lingüísticos vinculados con la región, el género, las características socioeconómicas y otras fuentes de identidad, incluidas aquellas relacionadas con el lenguaje ideológico. Asimismo, Georgakopoulou (2006) propone que hay que enfocarse en el surgimiento de identidades en el discurso, particularmente en sitios interactivos, donde pueden presentar una multiplicidad de significados. 


\subsection{Sobre los recursos de atenuación en narraciones}

La atenuación es definida por Briz (2007) como una estrategia lingüística que utilizan los hablantes para distanciarse de aquello que señalan, con el propósito de conseguir un acercamiento social con otros sujetos y que, como tal, varía según el contexto situacional de la interacción (Briz y Albelda, 2013). Cestero y Albelda (2012) también han propuesto que la atenuación varía socialmente. Por su parte, González Riffo (2017) propone que, para el caso de las narraciones, la atenuación "constituye un recurso de evaluación mediante el cual los hablantes validan sus historias como verdaderas y creíbles, lo que permite a los narradores equilibrar la tensión entre la reportabilidad y la credibilidad” (p. 78).

Los trabajos de González Riffo y Guerrero González (2017) y González Riffo (2017) aluden a la posibilidad de organizar los recursos de atenuación presentes en narraciones de experiencia personal en ejes pragmáticos: Veracidad, Certeza y Estima. El primero permite atenuar qué tan real es aquello que se dice, ya sea por parte del hablante mismo o de su alocutor. Certeza, por su parte, atiende a la modalización de la seguridad por parte del sujeto en relación con el contenido de las emisiones proferidas; es útil para que los narradores den cuenta de detalles de cuya especificidad no están seguros, pero aun así los consideran lo suficientemente relevantes como para comentarlos. Finalmente, Estima implica la atenuación de las evaluaciones ${ }^{2}$ emitidas, por lo tanto, se trata de recursos que extreman el componente interpersonal en la interacción. Los recursos de atenuación presentes en la 'orientación' narrativa se ubicarían, consecuentemente, en el eje de Certeza y corresponderían a ‘desfocalizadores' (cf. 3.4. de González Riffo, 2017).

Para llevar a cabo esta propuesta, los autores se basan en que (1) para Labov (2013), en el estudio de las narraciones de experiencia personal se aplica el principio de credibilidad, es decir, partir de la base de que aquello que se narra es real y, por ende, creíble. Dicho principio genera una tensión con la reportabilidad de la narración, lo que obliga al hablante a aplicar recursos verbales y no verbales, cuyo propósito es hacer su historia más real y, así, conservar su estatus de narrador veraz, y (2) en la interacción, los sujetos resguardan su imagen. En este sentido, son conscientes de qué es lo que la comunidad valora como una actitud positiva. En

\footnotetext{
${ }^{2}$ La evaluación corresponde, según Labov y Waletzky (1967) y Labov (2013), a todos los segmentos discursivos que son empleados por el narrador para validar su narración, es decir, para dejar en claro cuál es la razón de ser de su relato y su meta al narrarlo. La evaluación marca la parte central o informativa de un relato, aunque puede ser transversal a este.
} 
particular, uno de estos elementos es el hecho de presentarse como una persona honesta, lo que incita a evadir la mentira.

A partir de lo anterior, se puede precisar que la narración de experiencia personal constituye un discurso en que uno o más narradores relatan una experiencia integrando significados ideativos que son evaluados. Asimismo, los hablantes utilizan recursos que permiten evaluar la reportabilidad y credibilidad de la historia. La narración, a la vez, sirve de medio que relaciona al hablante con su oyente, quien, asimismo, evalúa y juzga lo que le es narrado. Finalmente, entre narrador e interlocutor existe una distancia interpersonal que, en la interacción, configura una relación interpersonal que se puede mantener siempre y cuando los interactuantes formulen actos de habla que mantengan la armonía. En consecuencia, se postula que la atenuación corresponde a uno de aquellos mecanismos utilizados para equilibrar la reportabilidad de la narración y su credibilidad, así como la de su narrador con el objetivo de resguardar su imagen positiva en beneficio de la armonía interpersonal.

\section{Metodología 3}

\subsection{Corpus}

En este estudio se analizan 90 narraciones de experiencia personal de hablantes de Santiago de Chile, cuyas edades oscilan entre los 35 y los 54 años. De dichos relatos, 36 narraciones son contadas de manera individual y corresponden al corpus de entrevistas sociolingüísticas del Proyecto Para el Estudio del Español de España y América de Santiago de Chile (PRESEEA-SA), mientras que las 54 restantes corresponden a narraciones coconstruidas (o conversacionales o diádicas) y son parte del proyecto FONDECYT 11150007.

Las narraciones del PRESEEA-SA se obtienen a partir de una entrevista sociolingüística, para lo cual se aprovecha el curso de Sociolingüística de la Licenciatura en Lengua y Literatura Hispánicas y Lengua y Literatura Inglesas de la Universidad de Chile. Por su parte, las narraciones co-construidas o diádicas se obtienen a partir del diseño narrativo para el estudio autobiográfico y de tópico y son grabadas audiovisualmente. En ambos casos, se utiliza transcripción con ortografía convencional.

\footnotetext{
3 La metodología empleada en esta investigación reproduce en buena medida la utilizada en el proyecto FONDECYT 11150007 y en las investigaciones derivadas de él.
} 


\subsection{Población y muestra}

Todos los informantes tienen edades entre 35 y 54 años, dados los hallazgos de Guerrero (2014a), quien, en su tesis doctoral, concluye que este grupo etario es el más sensible a la variación. Según Blas Arroyo (2005), se trata, además, de un grupo en cuya etapa vital los sujetos pasan por un desarrollo laboral pleno y ya tiene los patrones adultos de variación.

Finalmente, seguimos las sugerencias de Moreno Fernández (1990) y Hernández Campoy y Almeida (2005) en cuanto al tamaño muestral. Las cuotas quedan organizadas por afijación uniforme (López Morales, 1994), considerando las variables sociales grupo socioeconómico y sexo. La distribución de la muestra se sintetiza en las tablas 1 y 2.

Tabla 1. Tabla de distribución de informantes de PRESEEA-Santiago de Chile: narraciones individuales.

\begin{tabular}{llll}
\hline $\begin{array}{l}\text { Grupo } \\
\text { socioeconómico }\end{array}$ & Mujer & Hombre & Totales \\
\hline Bajo (B) & 6 & 6 & 12 \\
Medio (M) & 6 & 6 & 12 \\
Alto (A) & 6 & 6 & 12 \\
Totales & 18 & 18 & 36
\end{tabular}

Tabla 2. Tabla de distribución de los informantes del Proyecto FONDECYT No 11150007: narraciones conversacionales.

\begin{tabular}{lllll}
\hline $\begin{array}{l}\text { Grupo } \\
\text { socioeconómico }\end{array}$ & Hombre-Hombre & Hombre-Mujer & Mujer-Mujer & Totales \\
\hline Bajo (B) & 6 & & & \\
Medio (M) & 6 & 6 & 6 & 18 \\
Alto (A) & 6 & 6 & 6 & 18 \\
Totales & 18 & 6 & 6 & 18 \\
\hline
\end{tabular}

La estratificación de nuestra muestra se basa en el sistema del Proyecto PRESEEA, la que contempla el nivel de instrucción de los hablantes y los organiza en básico (bajo), secundario (medio) y superior (alto). El corpus de narraciones diádicas también utiliza este método; sin embargo, ambas estratificaciones se complementan con la estratificación empleada 
en el marco del proyecto de Estudio Sociolingüístico del Español de Chile (ESECH), el que considera una escala de estatus socioeconómico basada en las variables nivel educacional, categoría ocupacional y comuna de residencia (San Martín y Guerrero, 2015).

\subsection{Procesamiento analítico}

Si bien en otros estudios variacionistas que también tienen la atenuación como objeto de investigación se considera el acto de habla como unidad lingüística de análisis (cf. Cestero, 2011), en nuestra pesquisa utilizamos la cláusula ${ }^{4}$. Además, con el propósito de neutralizar la extensión del relato, que podría estar relacionada con la utilización de un mayor número de recursos de atenuación ${ }^{5}$, proponemos un índice que evidencia el promedio del número de ocurrencias de un tipo específico o de varios recursos de atenuación por cada cláusula presente en el relato (total de atenuadores divido por total de cláusulas, con respecto a cada narración). Así, un índice de 1.1 refleja que, por cada cláusula, el hablante suele ocupar un recurso de atenuación.

Los datos fueron procesados con el software de análisis estadístico R (R Core Team, 2015; Lawrence, 2015; Wicham, 2009). En primer lugar, y para controlar las diferencias entre las extensiones de las emisiones en cada relato (ya sea diádico o individual), se procedió a registrar y clasificar la cantidad de recursos de atenuación observados en cada narración, para luego dividirla por el total de cláusulas observado para cada registro particular. Esto permitió contar con una medida comparable entre los registros estudiados. Posteriormente, se efectuaron diferentes pruebas de Anova factorial entre sujetos tomando como variable dependiente: a) el total de los recursos de atenuación registrados, convertido en el índice propuesto, en las orientaciones narrativas del corpus convertido y b) el desglose de cada tipo de recurso de atenuación orientacional. Estas pruebas fueron efectuadas tanto en las narraciones individuales como en los registros diádicos. Como variables independientes se ingresaron el nivel

\footnotetext{
${ }^{4}$ Entendemos cláusula como una estructura en que se combinan tres tipos de significados (Halliday, 1985): uno ideacional, uno interpersonal y uno textual, los que permitirían a los hablantes construir tanto su experiencia como también las relaciones sociales que negocian por medio de ondas semióticas (Martin y White, 2005).

${ }^{5}$ Por ejemplo, una narración que consiste en diez cláusulas puede presentar siete recursos atenuantes (discurso atenuado), mientras que otra de solo cinco cláusulas podría estar constituido de tres recursos (discurso + atenuado). Si bien el primero presenta una mayor ocurrencia de mecanismos, es el segundo el que está más atenuado, puesto que consideramos la extensión de la misma narración. Así, emulamos lo realizado por otras investigaciones que también han tenido este fenómeno como objeto de estudio, reemplazando al acto de habla como unidad lingüística de análisis.
} 
socioecónomico y el sexo de los informantes, lo que generó anovas factoriales entre sujetos de 3 x 3 (diadas) y 3 x 2 (narraciones individuales). Se inspeccionaron además los efectos aislados de las variables sexo y nivel socieoconómico mediante pruebas de Anova unifactorial sobre las variables dependientes anteriores. Al igual que en el caso de las pruebas multifactoriales, se realizaron los análisis tanto en las diadas como en los registros individuales.

\section{Análisis, presentación y discusión de los resultados}

Para facilitar la lectura de esta sección del trabajo, hemos dividido este apartado en tres subsecciones, a saber, la que relaciona la presencia de recursos de atenuación con los recursos de orientación extratemática (Guerrero 2014b), la que determina la sensibilidad a la variación de los recursos de atenuación en orientación narrativa en relación con los factores sexo y grupo socioeconómico de los hablantes y, por último, la que compara el uso de los recursos de atenuación según el tipo de narración, individual o conversacional.

4.1. Relación de la presencia de recursos de atenuación con los recursos de orientación extratemática

Los recursos de atenuación presentes en la 'orientación' narrativa corresponden a lo que González Riffo (2017) denomina 'desfocalizadores'. Los desfocalizadores corresponden a unidades léxicas que difuminan los límites semánticos de categorías o representaciones concebidas por los hablantes. Estos elementos equivalen a partículas que permiten a los informantes no comprometerse en su totalidad con la información proporcionada, ya sea porque no recuerda la particularidad de aquello que narra o bien porque duda parcial o totalmente de una idea, aunque prefieran modificarla de igual forma. El uso de este recurso correspondería a lo que en Albelda et al. (2014) se conceptualiza como segunda función pragmática de los atenuadores, en la que los informantes buscan prevenir efectos perlocutivos inconvenientes.

Como su ubicación en el discurso es previa a elementos bien precisos y objetivos como datos numéricos correspondientes a fechas u horarios, o características particulares sobre algo-, en la estructura narrativa se utilizan fundamentalmente en las orientaciones, entendidas como elementos que hacen referencia a un tiempo, a un lugar, a personas y a conductas 
esperadas dentro de la narración (Labov y Waletzky, 1967; Labov, 2013). En esta línea, es importante el aporte de Guerrero (2014b), quien, como ya precisamos, a partir de los aportes de Labov y Waletzky (1967) y Labov (1972), propone nueve categorías de orientación, las que podríamos organizar, según los intereses de este estudio, en dos macrocategorías: aquellas que especifican un dato y otras que describen un elemento presente en la narración.

En ese contexto, con el propósito de precisar los datos que ofrecemos luego, incluimos tres casos en que los hablantes difuminan aquello que señalan, uno para especificaciones y dos para descripciones: desfocalización de tiempo y de lugar, desfocalización de rasgo y desfocalización de procesos y eventos, respectivamente. Mientras la primera responde a lo que Guerrero (2014b) conceptualiza en dos categorías, denominadas especificación de tiempo y especificación de lugar, en este estudio optamos por agruparlas en solo una para comprender cómo los hablantes presentan las coordinadas témporo-espaciales en las que se desarrolla lo narrado. Por su parte, las dos categorías restantes responden a descripciones y se diferencian, en específico, en si lo que matizan son objetos/lugares/momentos -descripciones de un algo- o procesos -descripciones de un como-. A continuación, ofrecemos ejemplos de la primera modalidad:

(1) SCHI_H21_013: "bueno eran como las cinco y media de la mañana"

(2) H-H03M: "y pasada la bencine- la bencinera que quedaba como a treinta kilómetros"

Como se observa, (1) y (2) corresponden a casos en que se aprecia una desfocalización de tiempo o lugar. En particular, lo destacado con negrita en (1) desfocaliza el tiempo del relato al presentar el contexto temporal en que ciertos acontecimientos tuvieron lugar, mientras que en (2) se opta por matizar una distancia geográfica. Al igual que en estos dos casos, es recurrente que se utilice este recurso cuando los informantes dan cuenta de un dato numérico, probablemente, por tratarse una materia específica que los hablantes prefieran atenuar con el fin de evitar errores en la información entregada. Asimismo, es frecuente el uso del adverbio <como> en su uso atenuador con valor aproximativo (cf. Panussis, 2016). Incluso, sobre este último recurso Poblete, Pons y Samaniego (2000) agregan que en el español culto del español de Chile es más frecuente en las producciones de jóvenes y mujeres. Los ejemplos que siguen son de descripciones: 
(3) SCHI_M23_091: "en vez de empapelado como en género / o quizás era papel"

(4) M-M03A: "en todos los ámbitos. Era como bien buena consejera"

(5) SCHI_M23_096: "prefiero quedarme con la duda y salir arran- o sea hablando corriendo casi"

(6) H-M05M: "no sé yo sentí muy largo porque como que llegaba gente y al principio no nos daban comida"

En (3) se observa lo que Guerrero (2014b) denomina una orientación por descripción objetiva de un objeto, mientras que (4) se trataría de una evaluación. Independiente al carácter subjetivo que entregan las informantes en ese último enunciado, lo que se realiza allí es justamente una descripción de una persona. Por su parte, lo ennegrecido en (5) permite dudar de si efectivamente la acción que se realizó fue correr o si solo se trató de una caminata rápida con características de una corrida; mientras que lo ennegrecido en (6) alude a un evento particular que es la llegada de gente a un lugar determinado. En este sentido, los atenuadores de (5) y de (6) cumplen con modificar un proceso y no un objeto o una persona.

Cabe destacar que este recurso es considerado dentro de la red sistémica del Modelo de Valoración (cf. Martin y White, 2005) como el subsistema de FOCO, dentro del sistema de GRADACIÓN. Esta consideración denota que el recurso de desfocalización revela efectivamente el imaginario individual y colectivo de los sujetos, así como también constituye un potencial para que los hablantes intensifiquen o mitiguen la subjetividad expresada en su discurso. De la misma forma, en la revisión del corpus hemos observado que estos recursos se acompañan de otros que fortalecen el matiz proferido. En concreto, y aunque no de forma obligatoria, suelen estar acompañados, por ejemplo, de verbos modales, de explicaciones o de justificaciones.

4.2. Comparación del uso de los recursos de atenuación según el tipo de narración, individual o conversacional

La consideración del tipo de construcción de la narración, sea individual o conversacional, es importante en tanto podría revelar si el contexto de interacción propicia el uso de recursos de atenuación dentro de las orientaciones narrativas. En total, en el corpus se identificaron 265 atenuadores desfocalizadores dispuestos en orientaciones narrativas, 95 de las cuales son de narraciones individuales y 170 de narraciones conversacionales conversacionales. Al considerar este factor los resultados de la investigación son los que se ilustran en el gráfico que sigue. 
Gráfico 1. Relación entre la media de recursos de atenuación por cláusula en orientaciones narrativas y tipo de narración.

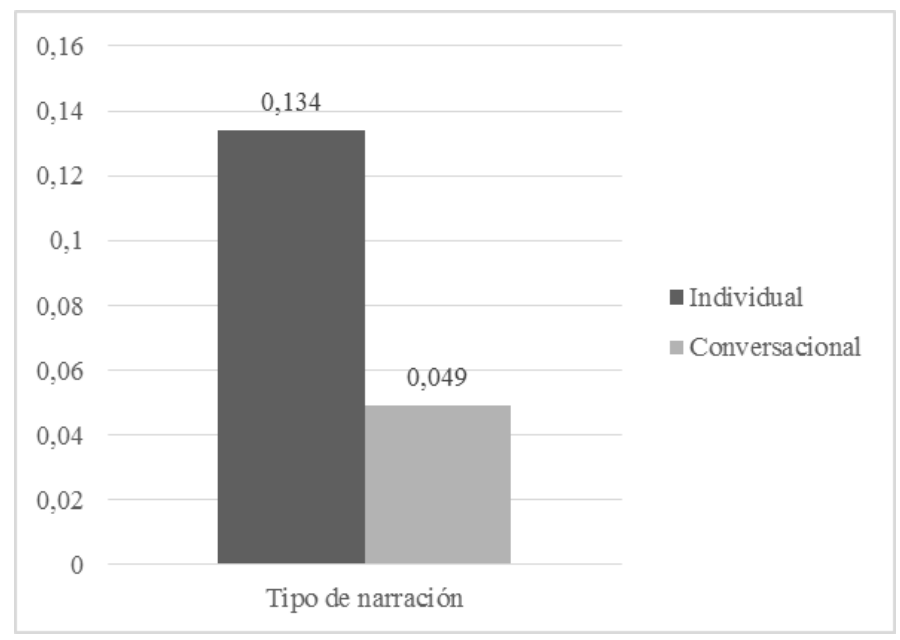

Como se puede observar, los hablantes atenúan los detalles de las orientaciones narrativas en mayor medida cuando se trata de narraciones construidas individualmente. Los datos son estadísticamente significativos con un valor $\mathrm{p}=0,00$. Este hallazgo se repite al revisar la propuesta de 4.1. sobre la naturaleza de la información a la que se remite, tal como se sintetiza en la siguiente tabla.

Tabla 3. Relación entre la media de recursos de atenuación por cláusula en orientaciones narrativas desglosadas y tipo de narración.

\begin{tabular}{lccc}
\hline & Individual & Conversacional & p \\
\hline Tiempo/Lugar & 0,04 & 0,68 & 0,00 \\
Rasgo & 0,06 & 0,63 & 0,01 \\
Proceso & 0,02 & 0,25 & 0,05 \\
\hline
\end{tabular}

Estos resultados se podrían relacionar con las descripciones de Briz y Albelda (2013) respecto con que, en contextos de mayor inmediatez comunicativa y mayor coloquialidad, menor es la ocurrencia de recursos de atenuación discursivos. Así, la narración conversacional se caracterizaría por este mismo principio, mientras que la narrativa individual podría describirse como una instancia en la que los hablantes sienten más amenazada su imagen, en términos de Goffman $(1959,1967)$. 
Además, es posible concebir que la atenuación de orientaciones narrativas en los relatos conversacionales provoca un efecto dialógico en la construcción narrativa, es decir, puede funcionar como una suerte de invitación a que el co-narrador complete o precise la información, también con el objetivo de proteger la imagen frente a la incertidumbre. En consecuencia, será interesante revisar cómo los factores sociales se relacionan con este fenómeno en dichas historias.

4.3. Sensibilidad a la variación de los recursos de atenuación en orientación narrativa en relación con los factores sexo y grupo socioeconómico de los hablantes

En lo que sigue, presentamos los resultados que atienden a la relación entre el uso de recursos de atenuación en orientaciones narrativas y los factores sociales sexo y grupo socioeconómico. Es necesario precisar que la mayor parte de los resultados no es estadísticamente significativa, pero que refleja ciertos patrones de comportamiento recurrentes y fuertemente descritos en otras variedades del español.

En cuanto al factor sexo, para las narraciones individuales las relaciones son las que se presentan en el gráfico 2.

Gráfico 2. Relación entre la media de recursos de atenuación por cláusula en orientaciones narrativas y factor sexo en narraciones individuales.

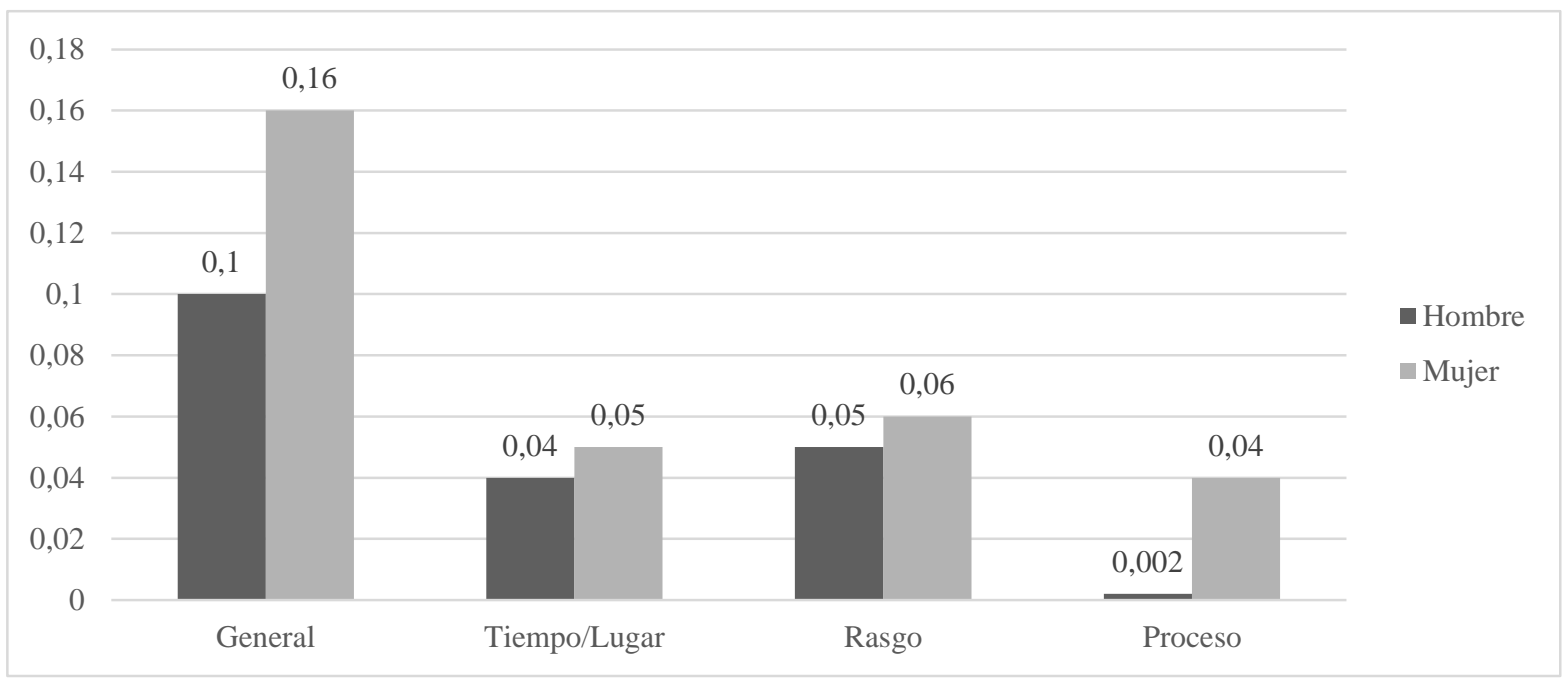


De forma transversal, son las narraciones de las mujeres las que presentan una mayor ocurrencia del fenómeno en estudio. Además, se concluye que la diferencia se acentúa principalmente en las orientaciones que aluden a procesos o eventos. Es este elemento el único que muestra resultados estadísticamente significativos $(\mathrm{p}=0,01)$.

En lo que respecta a las narraciones co-construidas por dos hablantes, cuyos datos se sintetizan en el gráfico 3, se aprecia que de forma transversal son las elicitadas por parejas de distinto sexo las que presentan una mayor ocurrencia del fenómeno.

Gráfico 3. Relación entre la media de atenuadores por cláusula en orientaciones narrativas y factor sexo en narraciones conversacionales.

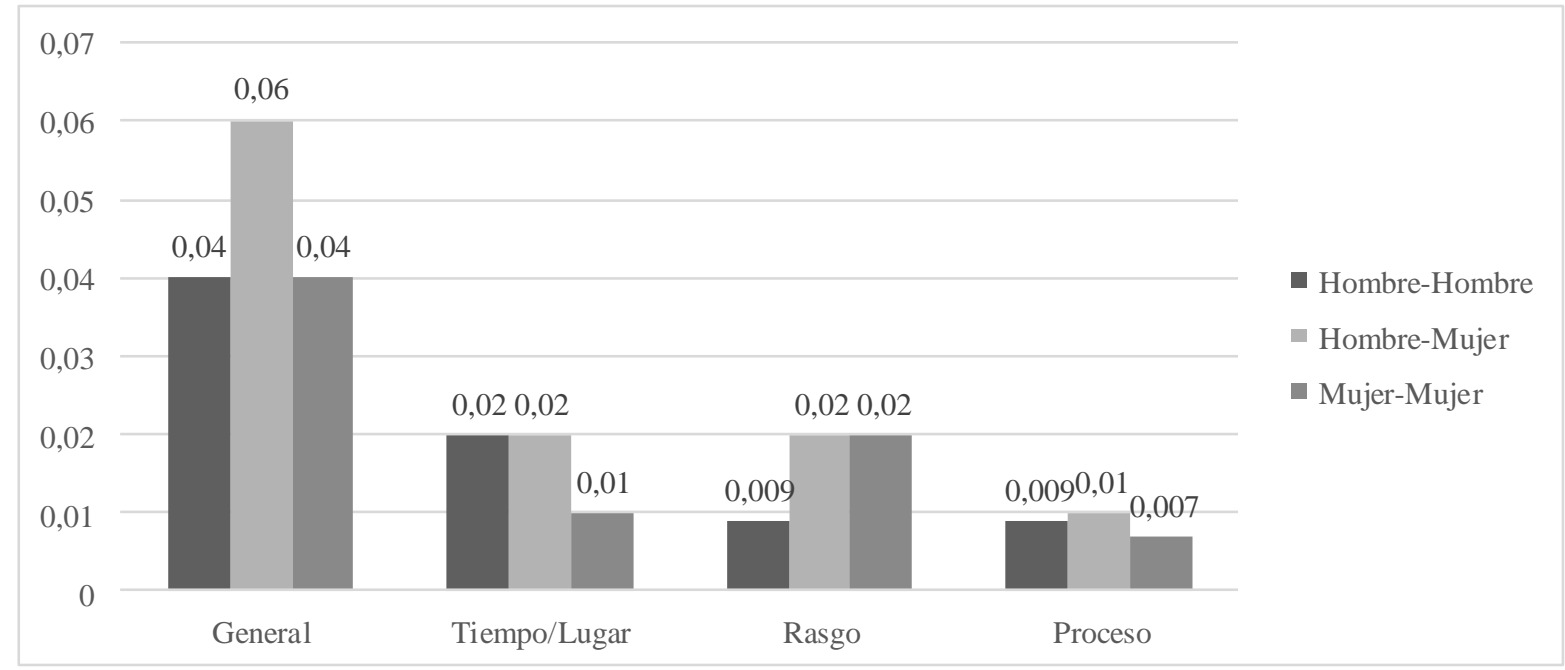

Si bien se podría pensar que la mujer es la que emplea más recursos de atenuación en orientaciones narrativas, dadas las descripciones que la sitúan como más empática (cf. Tannen 2011; 2017), esta idea se puede desechar al momento de considerar que para los casos de tiempo/lugar las medias presentes en las narraciones hombre-mujer se igualan a las de los relatos hombre-hombre, y que para las que aluden a rasgo se igualan a las de mujer-mujer. La diferencia, entonces, se remite principalmente a los datos de proceso, en cuyo caso hemos demostrado que son las mujeres quienes, de forma individual, propician el empleo de recursos de atenuación (cf. Gráfico 2). Pese a esto, son las narraciones construidas por dos mujeres las que registran la menor cantidad de recursos de atenuación (con medias de 0,04 para el 
panorama general, 0,01 para las de tiempo/lugar, 0,02 para las de rasgo y 0,007 para las de proceso).

En cuanto al factor grupo socioeconómico, presentamos el gráfico que sintetiza la información relativa a las narraciones individuales.

Gráfico 4. Relación entre la media de recursos de atenuación por cláusula en orientaciones narrativas y grupo socioeconómico en narraciones individuales.

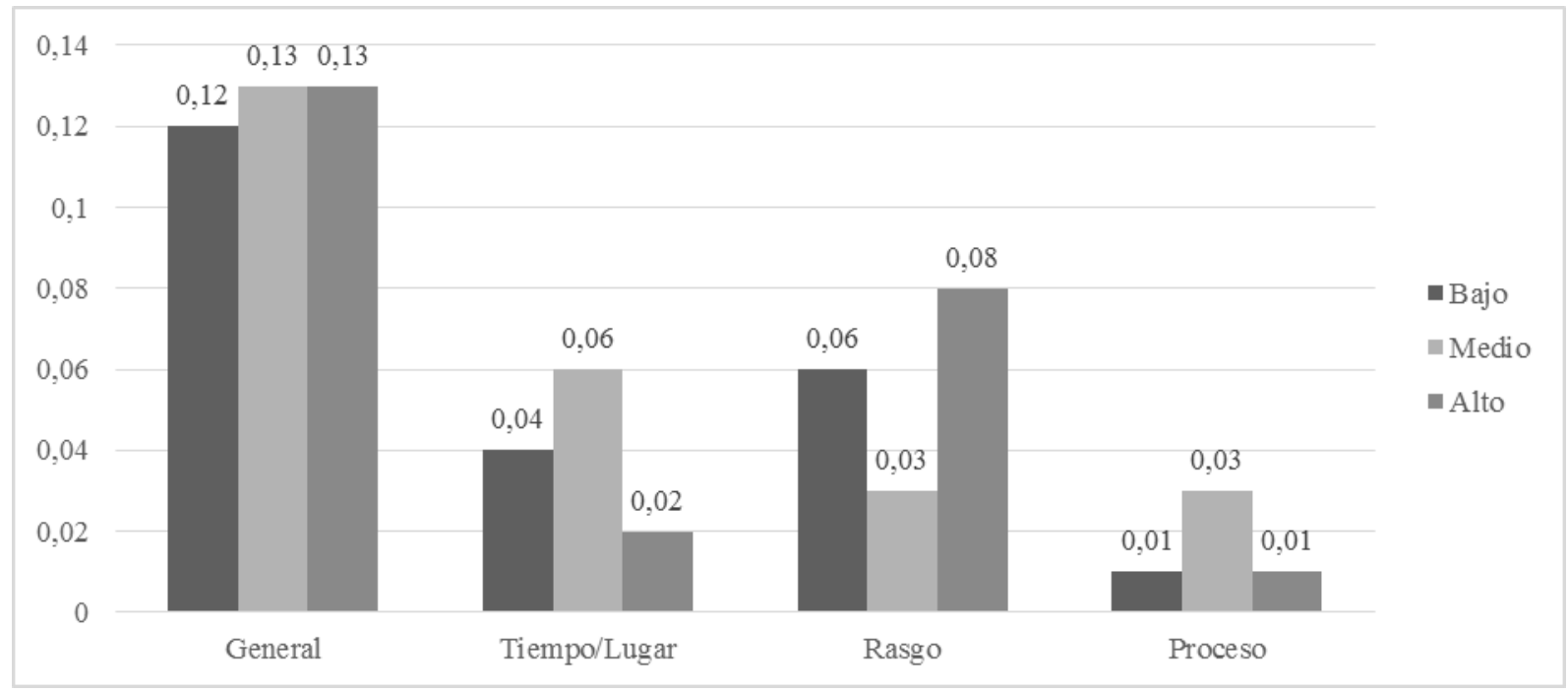

En el panorama general, se observa que no existen mayores diferencias en el empleo de recursos de atenuación por los tres grupos en estudio. Sin embargo, los datos específicos del tipo de orientación presentan algunos datos interesantes a nivel descriptivo, aunque no estadísticamente significativos. Concretamente, es relevante que las coordenadas de tiempo/lugar y proceso incluyan más recursos de atenuación cuando corresponden al grupo medio de la escala social, mientras que el comportamiento sea inverso en los datos correspondientes a rasgos, probablemente porque estos muchas veces se asignan para caracterizar entes animados, lo que podría ser más susceptible a juicios que decanten en actos descorteses. Insistimos en que se trata de hallazgos cuya significación estadística, para esta muestra, no pudo confirmarse, pero que son altamente interesantes desde el punto de vista de la tendencia porcentual que presentan. 
Gráfico 5. Relación entre la media de recursos de atenuación por cláusula en orientaciones narrativas y grupo socioeconómico en narraciones

conversacionales.

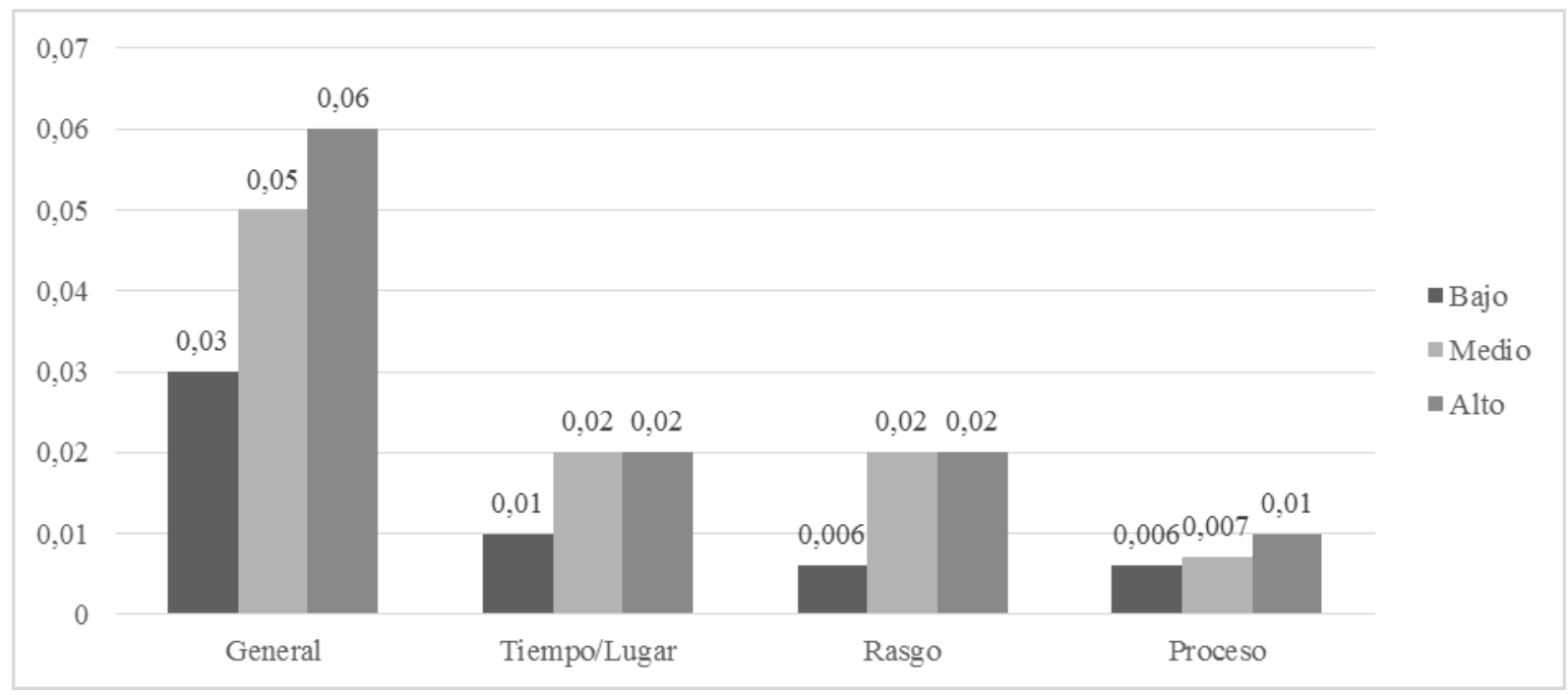

En efecto, y pese a que los datos no son estadísticamente significativos ( $\mathrm{p}=0,384$ para el panorama general; $\mathrm{p}=0,94$ para las orientaciones de tiempo/lugar; $\mathrm{p}=0,16$ para las de rasgo; $\mathrm{y}$ $\mathrm{p}=0,384$ para las de proceso), en las narraciones conversacionales se suelen acentuar las diferencias en contraste con los resultados evidenciados en los relatos individuales. En concreto, los hablantes de grupos socioeconómicos más altos atenúan más en el panorama general y en las orientaciones de procesos. Además, en cuanto a su distribución, se comportan de manera similar que los grupos medios y presentan más recursos de atenuación que los grupos bajos en la orientación que alude a tiempo/lugar y rasgos. Estas caracterizaciones, aunque son las restricciones correspondientes únicamente al ámbito de la estadística descriptiva, se apegan a los resultados de otras investigaciones que señalan que los hablantes de grupos socioeconómicos altos atenúan más, porque sienten más frecuentemente amenazada su imagen, por lo que ejecutan estrategias que les permitan aminorar tales efectos (cf. Molina Martos, 2015; Cestero, 2011). Dado que se trata de una tendencia, convendría profundizar en el análisis de la atenuación en interacción, con el propósito de verificar si en la variedad del español de Chile, los hablantes siguen los patrones de comportamiento pragmático que han descrito, para el español de España, las autoras antes citadas. 


\section{Conclusiones}

Los principales hallazgos de esta investigación son los siguientes:

1. Los recursos de atenuación en la 'orientación' de narrativas orales corresponden a lo que González Riffo (2017) denomina ‘desfocalizadores', que corresponden a unidades léxicas que difuminan los límites semánticos de categorías o representaciones concebidas por los hablantes.

2. Los hablantes atenúan orientaciones narrativas con menor frecuencia cuando se narra junto a un co-narrador o, en otras palabras, en el marco de la narrativa conversacional. Se cree que esto se podría deber a que la presencia de otro participante que estuvo presente en la sucesión de eventos reportada podría ser suficiente para que la historia sea creíble, sin entonces afectar la reportabilidad de la historia. Además, se interpretan estos datos a la luz de los aportes de Briz y Albelda (2013), quienes señalan que en contextos de mayor coloquialidad e inmediatez comunicativa los hablantes atenúan, en general, menos que en otras circunstancias. En este sentido, se podría creer que la narración conversacional emula un ambiente de mayor distención.

3. Con respecto al factor sexo, se aprecia que las mujeres utilizan en mayor cantidad recursos de atenuación en las historias narradas individualmente. Para las narraciones conversacionales, por medio de la atenuación de la orientación, se propicia el diálogo con el co-narrador, muy probablemente, con el propósito de que este participe de manera activa en la construcción del relato, lo que no es posible en los relatos que se generan de manera individual en el contexto de la entrevista sociolingüística, caracterizada por ser antes que una conversación, una especie de monólogo.

4. En lo que concierne al factor grupo socioeconómico, si bien se trata solo de hallazgos descriptivos, los resultados siguen en líneas generales lo que la literatura sobre el tema investigado ha descrito con anterioridad, esto es, que a medida que se asciende en la escala social, el fenómeno de la atenuación se ve mayormente presente. Se trata de una conclusión que muestra, por lo menos, una tendencia en el comportamiento de los hablantes y que, al no presentar, para la variedad del español de Chile, resultados estadísticamente significativos, no permite realizar ningún tipo de generalización; sin 
embargo, creemos que al ser una tendencia observada en especial en las narraciones conversacionales, permite abrir nuevas líneas de investigación con base en nuevas hipótesis de trabajo relacionadas con la atenuación en interacción.

Basándonos en los resultados anteriores, destacamos la necesidad de revisar el posible vínculo entre el fenómeno en estudio y una mayor precisión en cuanto a la naturaleza de la información que se detalla en las orientaciones narrativas. De la misma forma, es interesante considerar la disposición de los recursos de atenuación, en general, en toda la estructura narrativa, como, por ejemplo, en las acciones complicantes. Por último, dado que estudios recientes han comenzado a reconocer la relevancia de la comunicación no verbal en el empleo de estas estrategias discursivas (Cestero, 2014, 2016; Guerrero, 2017b), consideramos fundamental complementar los datos expuestos en esta investigación con aquellos detalles no verbales que permitirían completar el panorama revisado.

\section{Bibliografía}

Albelda, Marta, Briz, Antonio, Cestero, Ana Kotwica, Dorota y Villalba, Cristina. "Ficha metodológica para el análisis pragmático de la atenuación en corpus discursivos del español (ES.POR.ATENUACIÓN)". Oralia 17 (2014): 7-62.

Bassols, Margarida y Torrent, Ana. Modelos textuales. Barcelona: Eumo-Octaedro, 2003.

Blas Arroyo, José Luis. Sociolingüística del español. Desarrollos y perspectivas en el estudio de la lengua española en contexto social. Madrid: Cátedra, 2005.

Briz, Antonio. "Para un análisis semántico, pragmático y sociopragmático de la cortesía atenuadora en España y América”. Lingüística Española Actual 29. 1. (2007): 5-40.

Briz, Antonio y Albelda, Marta. "Una propuesta teórica y metodológica para el análisis de la atenuación lingüística en español y portugués. La base de un proyecto en común (ES.POR.ATENUACIÓN)”. Onomázein 27.2. (2013): 288-319.

Cestero, Ana. "Las estrategias de atenuación: estudio sociolingüístico". Actas del IX Congreso Internacional de Lingüistica General. Valladolid, Universidad de Valladolid, 2011: 525-542.

Cestero, Ana. "Comunicación no verbal y comunicación eficaz". Estudios de Lingüística 28 (2014): 125-150.

Cestero, Ana. "La comunicación no verbal: propuestas metodológicas para su estudio". LinRed, 13 (2016). http://www.linred.es/numero13_2_monografico_Art1.html

Cestero, Ana y Albelda, Marta. "La atenuación lingüística como fenómeno variable". Oralia, 15 (2012): 77-124. 
De Fina, Anna y Johnstone, Barbara. "Discourse Analysis and Narrative". The Handbook of Discourse Analysis, eds. Debora Tannen, Heidi Hamilton y Deborah Schiffrin. Malden, Massachusetts: Wiley-Blackwell. 2015: 152-168.

Georgakopoulou, Alexandra. "Small and large identities in narrative (inter)action". Discourse and identity, eds. Anna De Fina, Deborah Schiffrin y Michael Bamberg. Cambridge: Cambridge University Press, 2006: 83-102.

Goffman, Erving. The Presentation of Self in Everyday life. New York: Anchor Books, 1959.

Goffman, Erving. Interactional Ritual: Essays on Face-To-Face Behavior. New York: Pantheon Books, 1967.

González Riffo, Javier. Estrategias de atenuación en narraciones de experiencia personal de hablantes de Santiago de Chile: un estudio sociopragmático. Tesis, grado de Magíster en lingüística con Mención en Lengua Española. Universidad de Chile, 2017.

González Riffo, Javier y Guerrero González, Silvana. "Estrategias de atenuación en narraciones conversacionales". Lengua y Habla 21(2017): 29-44.

Guerrero, Silvana. Variación discursiva en narraciones de experiencia personal en el español hablado en Santiago de Chile. Tesis, doctoral, Chile: Pontificia Universidad Católica de Chile, 2014a

Guerrero, Silvana. "Una propuesta de categorización de los rasgos variables de la orientación en narraciones de experiencia personal”. Boletín de Filología 49. 1. (2014b): 221-233.

Guerrero, Silvana. "La recolección de narraciones orales y su estudio en correlación con factores sociales: el valor de la entrevista sociolingüística”. Philologica Canariensia 23 (2017a): 79-89.

Guerrero, Silvana. "Los reguladores no verbales de inicio de secuencia estructural en narraciones conversacionales: un estudio sociopragmático". Pragmalingüística 25 (2017b): 267-285.

Guerrero, Silvana. Narración individual versus narración conversacional: análisis de las diferencias de superficie, (m.i.).

Halliday, Michael. An introduction to Functional Grammar. Londres: Edward Arnold, 1985.

Hernández-Campoy, Juan Manuel y Almeida, Manuel. Metodología de la investigación sociolingüística. Málaga: Editorial Comares, 2005.

Johnstone, Barbara. "Variation in discourse: Midwestern narrative style". American Speech 65. 3 (1990): 195-214.

Johnstone, Barbara. "Discourse analysis and narrative". Handbook of discourse analysis, eds. Deborah Schiffrin, Deborah. Tannen y Heidi Hamilton. Malden, MA: Blackwell, 2001: 635-649.

Johnstone, Barbara. "A new role for narrative in variationist sociolinguistics". Narrative Inquiry 1 (2006): 46-55.

Juzwik, Mary. "Spoken Narrative". The Routledge Handbook of Discourse Analysis. Routledge Handbooks in Applied Linguistics, eds. James Gee y Michael Handford. London: Routledge, 2012: 326-341.

Labov, William y Waletzky Joshua. "Narrative analysis". Essays on the Verbal and Visual Arts, ed. June Helm. Seattle: University of Washington Press, 1967: 12-44.

Labov, William. "The transformation of experience in narrative syntax". Language in the inner city. Studies in the Black English Vernacular. Filadelfia. University of Pennsylvania Press, 1972: 395-415. 
Labov, William. "Some further steps in narrative analysis". Journal of Narrative and Life History 7 (1997): 395- 415.

Labov, William. “Ordinary events". Sociolinguistic Variation: Critical Reflections, ed. Carmen Fought. Oxford: Oxford University Press, 2004: 31-43.

Labov, William. The Language of Life and Death. The Transformation of Experience in Oral Narrative. Cambridge: University Press, 2013.

Lawrence, Michael. Easy Analysis and Visualization of Factorial Experiments. R package version 4.3. 2015. https://CRAN.R-project.org/package=ez

López Morales, Humberto. Métodos de investigación lingüística. Salamanca: Ediciones Colegio de España, 1994.

Martin, Jim y White, Peter. The language of Evaluation. Appraisal in English. New York: Palgrave Macmillan, 2005.

Molina Martos, Isabel. "Estrategias de atenuación en el barrio de Salamanca de Madrid". Patrones sociolingüísticos de Madrid, eds. Ana Cestero, Isabel Molina y Florentino Paredes. Berna: Peter Lang, 2015.

Moreno Fernández, Francisco. Metodología sociolingüística. Madrid: Gredos, 1990.

Norrick, Nick. Conversational narrative. Saarland University: John Benjamins Publishing Company, 2000.

Panussis, Constanza. Análisis pragmático y sociolingüístico de las funciones del marcador como (que) en el habla de Santiago de Chile. Tesis, grado de licenciada en lengua y literatura hispánica con Mención en Lingüística. Universidad de Chile, 2016.

Poblete, Mario, Pons, Hernán y Samaniego, José Luis. "Fenómenos gramaticales y recursos modalizadores del enunciado en el español culto de Santiago de Chile". Onomázein, 5 (2000): 143-151.

R Core Team. R: A Language and Environment for Statistical Computing, 2015. http://www.R-project.org/

Riessman, Catherine. Narrative Methods for the Human Sciences. Sage Publications, Inc, 2008.

Soler, Sandra. Discurso y género en historias de vida. Una investigación de relatos de hombres y mujeres en Bogotá. Bogotá: Publicaciones del Instituto Caro y Cuervo, 2004.

San Martín, Abelardo y Guerrero, Silvana. "Estudio Sociolingüístico del Español de Chile (ESECH): recogida y estratificación del corpus de Santiago". Boletín de Filología 50. 1. (2015): 221-247.

Tannen, Deborah. That's not what I meant! New York: HarperCollins Publishers, 2011.

Tannen, Deborah. You're the only one I can tell. Inside the language of women's friendships. New York: Ballantine Books, 2017.

Thornborrow, Joanna. "Narrative Analysis". The Routledge Handbook of Discourse Analysis, Routledge Handbooks in Applied Linguistics, eds. James Gee y Michael Handford. London: Routledge, 2012: 51-65.

Wickham, Hadley. ggplot2: Elegant Graphics for Data Analysis. Dordrecht, New York: Springer, 2009. 